\title{
Visual Information Priming in Internet of Things: Focusing on the interface of smart refrigerator
}

\author{
Hyeonjin $\mathrm{Kim}^{1}$, Sangmee Lee ${ }^{1}$, and Donghee $\mathrm{Shin}^{1, *}$ \\ ${ }^{1}$ Department of Interaction Science, Sungkyunkwan University, 314 International Hall, 53 \\ Myeongnyun 3-ga, Seoul, Korea
}

\begin{abstract}
Internet of Things (IoT) supports an environment where users are able to interact and get information by sharing visual informational cues. The purpose of this study was to investigate what effect was made on the perceived usefulness, perceived ease of use, intention to use and selfefficacy about use of smart refrigerator when visual information related to the food and function is provided in advance in the interface. A data was collected from a between-subjects experiment $(\mathrm{N}=26)$ with an independent variable (priming visual information vs. non-priming). According to the experiment results, users who were primed about the visual cues perceived higher usefulness (PU), ease of use (PEoU), intention to use (IU), and self-efficacy (SE) toward the fridge compared to the users in a non-primed group. The study proposes theoretical and practical implications for designing food-related smart home appliances' interface and visual affordances.
\end{abstract}

\section{Introduction}

As information and the environment where objects are connected to the wireless network are shared, the objects inside households are also being evolved [1]. Smart refrigerator which applies various on-line network services provides better in-life object internet experience by supporting functions such as the temperature adjustment, conserved food management, grocery shopping, memo board and recipe. A display screen installed in the front of the product possesses potential value enlightening the convenience and efficiency of the product user [2]. However, while the information that the user should accept increases, the heuristic lacks about use of various functions and thus there are limitations in that the user use various functions voluntarily $[3,4]$. According to the system interface studies, it has been reported that this problem is caused by the conventional drop-down menu selection method.

Especially, since the various icons shown in the menu screen of the smart refrigerator have limitation in explain detailed functions that each of the sub-information contains, they obstruct the natural information search of the user when the user accesses the target information and there is complexity of use. In order to solve this problem, a study about

\footnotetext{
*Corresponding author: dshin@skku.edu
} 
new precipitating cue is needed which support easy and natural information search of the user and induce use of functions of the smart refrigerator. This study examines if selfefficacy increases when the information image that the refrigerator display proposes is exposed as the visual stimulus which reminds of the use of its functions. Also, this study has verified if the priming versus non-priming level of the corresponding information affects the reception of smart refrigerator technology.

\section{Theoretical Backgrounds}

\subsection{Information Priming}

A psychological concept of priming effects explains that whether the informational cue previously exposed to users affects their perception and recognition toward target stimulant and causes their behavioural changes [5]. According to the food advertisement studies, when consumers are exposed to the sense-related cues about food, their food consumption increases [6,7]. One of the food advertisements focuses on increasing instant satisfaction of consumers' sense by exposing them to the characteristic visual stimulus which provoked their appetite (e.g. hot vapor) [8]. When they are exposed to the stimulus in advance, complex physical behavior is vitalized and even in the cases where there is no special intention or when the consumer does not perceive appropriately the corresponding stimulus, such vitalization may occur due to the external stimulus [9]. Especially, consumers go through the priming process of various sensual stimulus (e.g. vision, hearing) when accepting food related information. In such cases, information recipients perceive the information (e.g. product benefits, ingredient) more effective and thus positive perception and attitude about products are formed [10]. Since smart refrigerator shows food and product related images based on on-line system, it can effectively use the food related image stimulus which induces use of refrigerator. Based on this rationale, we assume that when a smart refrigerator user is primed on the sensual stimulus, he or she can be induced to use the system voluntarily without a need to learn additionally about the use of submenu. Thus, the user who has been primed on visual stimulus will show positive reaction about use of instant system.

\subsection{Technology acceptance model (TAM) in effective interface}

The representative factors which affect the acceptance of new technology are perceived usefulness, perceived ease of use and intention to use technology. The perceived usefulness shall mean the perception that when the user uses the system, the individual outcome will improve, the perceived ease of use shall mean the belief that the user can use a particular system with no big efforts and the intention to use shall mean the acceptance of the user about a new information system based on the user's belief about a specific system [11].

According to the conventional studies, the TAM is appropriate to measure the positive effect of the visual image stimulus on the consumer. According to Hausman et al [12], there is a study result that when a user performs an on-line shopping task, the perception of usefulness improved according to if there were human factors (e.g. visual images). Especially, in the case of the internet based contents such as the digital archive resources, if effective interfaces such as user-friendly search tool are provided to the user on the interface, the perception of the user (perceived ease of use \& perceived usefulness) is affective positively [13]. Thus, this study intends to verify what effect the priming about visual cues has on the usefulness, predicted ease of use and intention to use when using interface of the smart refrigerator. 
- H1. The user's perceived usefulness (H1.1), perceived ease of use (H1.2) and intention to use (H1.3.) will be higher when the user is primed on the visual cues than when he is not primed.

\subsection{Self-Efficacy}

According to the social cognitive theory, self-efficacy is the belief that an individual's judgement of their ability to perform courses of action or achieve specific task. Such belief affects one's cognitive and behavioral outcome (i.e. motivation and performance) about use of a system [14]. According to Igbaria [15], it was said that when the user cannot access easily the necessary functions due to a system that he is not accustomed to, if help or interest is given about use of the system, the self-efficacy can be improved. Additionally, Hill and his colleagues [16] found that when self-efficacy is high, the technology acceptance about a new technology product rises. Thus, the self-efficacy is an important factor to affect user when accepting the new technology and thus it shall be measured along with the TAM model. In the case of the smart refrigerator, since its penetration rate is yet low, and thus there is a limitation in assessing the technology acceptance of the users who have not used a smart refrigerator, but since the self-efficacy also affects the prediction of use of the technology [17], it shall be used as a criteria for a user who has not used in person to assess the product.

- H2. The user's self-efficacy will be higher about use of smart refrigerator when the user is primed on visual cues than when he is not primed.

\section{Research Method}

The experiment participants were selected at random from the 26 applicants of students in their 20s through 30s who go to four year private universities in Seoul $(M=23.53, S D=$ 2.31). The recruitment of the participants was made against the people who have used a smart device for more than a year.

\subsection{Apparatus}

This study was conducted using the 13.3" screened laptop (LG 13ZD950 LX20K) in the laboratory. By excluding the characteristics appearance and design phase which represent specific brands, the study was made not affected by the manufacturer. The content used in the experiment was produced based on the LG Dios smart refrigeration, and the prototype interface was proposed (Figure 1). The experiment was composed in such a way that the experiment participants experience each given content according to the priming versus nonpriming level.

\subsection{Manipulation}

The priming level of the visual cues about use of smart refrigerator interface was divided into two experiment groups. (1) Home screen with no visual cue (2) home screen with visual cue priming. And difference of user's perception about each group was experimentally verified. In the visual cue with priming (Figure 2), in the left side, there is 
image of the materials kept at the refrigerator (e.g. mushroom, pork, clam etc) and in the right side, there is a image of recommended dish which can be made with the material kept. The image of the material kept in the refrigerator has been proposed in sliding method in turn with the text(e.g. the expiry date for the mush room is 2 days now.)

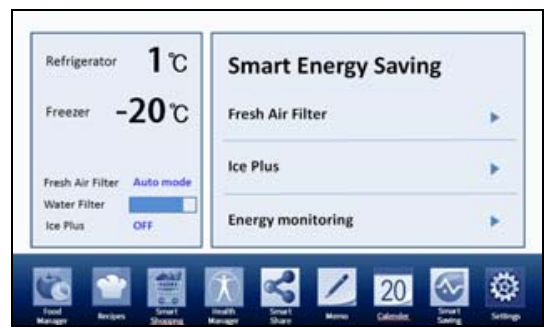

Fig. 1. Display interface (home screen) on

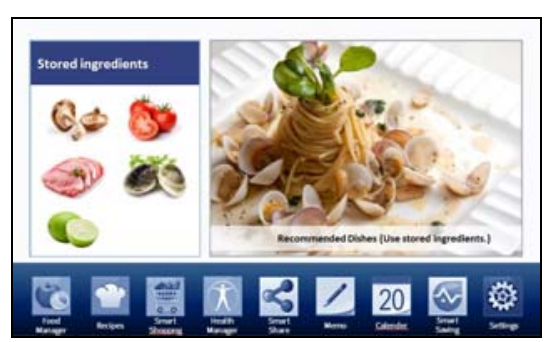

Fig. 2. A priming images on the display panel.

smart fridge

\subsection{Procedure}

The experiment participants were distributed at random at laptops of which the visual priming levels were differently set and they participated for 15 minutes. The experiment procedure was done in the order of the introduction to the experiment, the main experiment and questionnaire about visual priming cue after experiment. The subject group which was exposed to the smart refrigerator interface with visual cue priming was ordered to stare at the screen and follow the voice instruction. Each subject freely surveyed the functions (e.g. food management, recipe, shopping and energy management etc.) of the refrigerator using the icon on the bottom of the display. Other group subjects were exposed to directly to the icons on the bottom of the display without being proposed the image cue in advance. They surveyed the functions of the smart refrigerator freely just as the subjects with the visual cue priming did. After all the experiment was terminated, the experiment participants went through the questionnaire using a paper and a pencil.

\subsection{Measurement items}

As the perceived ease of use (Cronbach's $\alpha=.754$ ) of the Technology acceptance model [11], a total of three items including "I think that how to use the smart refrigerator is easy." And "To learn the functions of the smart refrigerator is easy to me" were measured, as the perceived usefulness(Cronbach's $\alpha=.854$ ), a total of three item including "I can do that task I want effectively when I use the smart refrigerator," "I think that use of smart refrigerator is valuable overall," and etc. were measured and as the intention of use (Cronbach's $\alpha=.877$ ), a total of three items including "I have the intention of using the various functions of the smart refrigerator" and "I will continuously use the various functions of the smart refrigerator" etc. were measured on a five-point Likert scale and all the scores were summed up and made an average.

As the self-efficacy [17] (Cronbach's $\alpha=.873$ ) a total of five items including "I will be able to use the smart refrigerator well even if there is no person explaining its functions" and "I will be able to use the functions well even if I have never used the smart refrigerator before" etc. were measured on a five-point Likert scale and the results were summed up and made average. 


\section{Results}

This study has used one-way analysis of variance (ANOVA) and analyzed in order to assess the effect of the priming of visual cue. It was verified that the main effect of the perceived usefulness according to the priming condition was higher in the primed condition $(M=4.692, S E=.099)$ than the non-primed condition $(M=3.674, S E=.099)$. It was verified that the main effect of perceived ease of use according to the priming condition was higher in the primed condition $(M=4.256, S E=.092)$ than in the non-primed condition $(M=3.712, S E=.092) .(F=17.544, p<.05, \eta 2=0.423)$. It was verified that the main effect of the intention to use according to the priming condition was higher in the primed condition $(M=4.654, S E=.105)$ than in the non-primed condition $(M=3.697, S E=$ $.105)$. $(F=41.428, p<.05, \eta 2=0.633)$ It was verified that the main effect of the selfefficacy according to the priming condition was higher in the primed condition $(M=3.385$, $S E=.168)$ than in the non-primed condition $(M=4.518, S E=.168) .(F=22.717, p<.05$, $\eta 2=0.487)$.

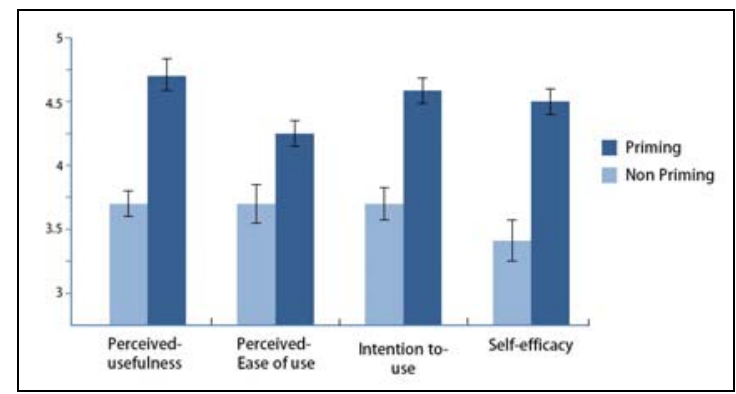

Fig. 3. Differences of each variable between the priming and non-priming level.

\section{Discussion and conclusion}

This research examines the perception and behavioral change of the user about the use of the smart refrigerator which is proposed later on according to the priming condition in advance of the visual information about use of the food and smart refrigerator. Based on this, thus study has proposed the smart refrigerator interface including the priming information based on the conventional smart refrigerator interface. According to the experiment result, a significant effect has been observed in terms of the perceived usefulness, perceived ease of use, intention to use and self-efficacy etc. according to the priming condition.

Based on the study result, two interpretations in two phases (TAM \& self-efficacy) are possible about visual information priming about use of smart refrigerator. First, as the study by Harris and colleagues has proposed, when the visual information about food has been primed for the user, the perception of the user about the product was more positive than in the non-primed condition of the visual information about food. Also, appetizing food images could be an attractive or appealing visual human factors in the technology use process enhanced the behavioral intention of the user. Further, it was revealed that when visual information related to the use of the smart refrigerator system has been proposed together, the visual priming affected the technology acceptance such as perceived ease of use, perceived usefulness and intention to use of the user.

Second, as in the $\mathrm{H} 2$, when the user has been primed about visual cue, the self-efficacy was very effective. As in the study asserted by Igbaria and his colleague [16], this was because the visual cue provided help and interest when the user used the technology that 
the self-efficacy was high. The users who have used smart refrigerator first time could find a cue which enhances their ability to use new function from watching expiration dates and recommended dishes. This can be seen as the fact that the prior priming of the visual information about use of smart refrigerator invoked provision of motive about use of smart refrigerator with the induction of interest or effective proposal of information and as a result of it, belief about the task performance ability about use of refrigerator system has been improved. This study has some limitations in future studies which will be conducted later on. First, since the experiment participants were in their 20s and 30s only and did not include people in their $40 \mathrm{~s}$ and $50 \mathrm{~s}$, there is a possibility that such participants can not represent the whole users of the smart refrigerator. However, in the case of the university students in their 20 s and 30 s, they have a high possibility of being potential users and they have possessed the experience of using smart machine, and thus it is considered that an ample result has been gained in measuring the smart refrigerator use experience. In future studies, based on these findings, the age and scope of the user layer will be expanded and more qualitative study will be conducted through in-depth behavioral study. Second, since the priming effect of the visual cue was measured only in the home screen currently, it will be needed to examine additional functions (e.g. food manager, smart shopping) in future studies. Through this, the priming of the visual cue can be deducted about effective functions and eventually, the users will be induced into use various functions of the smart refrigerator more. Although this study has been performed based on the smart refrigerator interface based on this content above, in the case of the smart appliances (e.g. smart oven) which have similar attributes based on the internet and which are related to food also, the method of proposing in advance the visual cue related to food and use of products can be applied and the use of smart appliances can be made automatically activated.

\section{References}

1. H. H. Lee, A. M. Fiore, J. Kim, International Journal of Retail \& Distribution Management, 34, 8 (2006)

2. D. Uckelmann, M.Harrison, F. Michahelles. Springer Berlin Heidelberg. (2011)

3. L. Swan, A. S. Taylor). CHI'05 extended abstracts on Human factors in computing systems, ACM. (2005)

4. P. Y. P. Chi, J. H. Chen, S. Y. Liu, \& H. H. Chu, Interaction Platforms and Techniques. (Springer Berlin Heidelberg, 2007)

5. M. Rothensee. (Springer Berlin Heidelberg 2008)

6. D. Logan, Cognitive psychology, 12, 4 (1980)

7. D. FEDOROFF, J. Polivy, C. P. Herman, Appetite, 28, 1(1997)

8. L. Terrenghi. (Springer Berlin Heidelberg, 2005)

9. R.Loewen, P. Pliner, Appetite, 32, 3 (1999)

10. A. Dijksterhuis, J. A. Bargh, Advances in experimental social psychology, 33, (2001)

11. J. L. Harris, J. A. Bargh, K. D. Brownell, Health psychology, 28,4 (2009)

12. F. D. Davis, Perceived usefulness, perceived ease of use, and user acceptance of information technology. MIS quarterly, (1989)

13. A. V. Hausman, J. S. Siekpe, Journal of Business Research, 62, 1 (2009).

14. J. C. Hong, M. Y. Hwang, H. F. Hsu, W. T. Wong, M. Y. Chen, Computers \& Education, 57, 3 (2011)

15. Bandura, Psychological review, 84, 2 (1977)

16. M. Igbaria, J. Iivari, Omega, 23, 6 (1995)

17. T. Hill, N. D. Smith, M. F. Mann, Journal of applied psychology, 72, 2 (1987)

18. Bandura, American psychologist, 37, 2 (1982) 